\title{
Proto-Calcite and Proto-Vaterite in Amorphous Calcium Carbonates**
}

\author{
Denis Gebauer,* Philips N. Gunawidjaja, J. Y. Peter Ko, Zoltán Bacsik, Baroz Aziz, Lijia Liu, \\ Yongfeng Hu, Lennart Bergström, Cheuk-Wai Tai, Tsun-Kong Sham, Mattias Edén, and \\ Niklas Hedin*
}

Crystallization of calcium carbonate, typically, progresses sequentially via metastable phases. Amorphous $\mathrm{CaCO}_{3}$ (ACC) generally forms initially, both in vitro and in vivo, and is the precursor of the predominant anhydrous polymorphs (calcite, aragonite, and vaterite) ${ }^{[1-1,1]}$ A new picture of the crystallization of calcium carbonate is emerging, which involves transformations of clusters to ACC and eventually to crystalline polymorphs. ${ }^{[14,15]}$ This stepwise manner has implications for the understanding of biomineralization ${ }^{[16]}$ and of crystallization. ACCs that contain additives display order over atomic length scales that are related to crystalline polymorphs; ${ }^{[1-3]}$ ACC synthesized at high supersaturation

[1] Dr. D. Gebauer, Dr. Z. Bacsik, B. Aziz, Prof. L. Bergström, Dr. N. Hedin

Department of Materials and Environmental Chemistry Materials Chemistry Unit, Stockholm University

10691 Stockholm (Sweden)

Fax: $(+46)$ 8-152-187

E-mail: denis.gebauer@mmk.su.se niklas.hedin@mmk.su.se

Homepage: http://www.mmk.su.se

Dr. P. N. Gunawidjaja, ${ }^{1+]}$ Dr. M. Edén

Department of Materials and Environmental Chemistry

Physical Chemistry Unit, Stockholm University

10691 Stockholm (Sweden)

Dr. J.Y. P. Ko, ${ }^{\mathrm{H}+}$ L. J. Liu, Prof. T.-K. Sham

Department of Chemistry, The University of Western Ontario London, ON N6A 587 (Canada)

Dr. Y. F. Hu

Canadian Light Source inc., University of Saskatchewan

Saskatoon, SK S7N OX4 (Canada)

Di. C.-W. Tai

Department of Materials and Environmental Chemistry, Structural and Inorganic Chemistry Unit, Stockholm University, 10691 Stockholm (Sweden)

[†] These authors contributed equally to this manuscript.

[각] This work was supported by the Swedish Research Council (VR), Ákesson Foundation, Natural Science and Engineering Research Council of Canada, Canada Research Chairs program, Ontario Innovations Trust, Canada Foundation for Innovation, National Research Council Canada, Canadian Institute of Health Research, and the University of Saskatchewan. D.G. and P.G. are supported by postdoctoral grants of the Wenner-Gren Foundations and the Carl Trygger Foundation, respectively. The Knut and Alice Wallenberg Foundations are acknowledged for an equipment grant for the NMR probehead and for electron microscopy facilities. We thank P.-E. Persson, T. Regier, and J. Weber for experimental help and $\mathrm{H}$. Cölfen and the reviewers for helpful suggestions. levels without additives, ${ }^{[17-20]}$ on the other hand, show no distinct short-range order. ${ }^{[21.22]}$

Herein, we analyze proto-crystalline features of two amorphous intermediates, $\mathrm{ACCl}$ and $\mathrm{ACCII},{ }^{[15]}$ and discuss their relevance for crystallization of $\mathrm{CaCO}_{3}$. We rationalize the identification of ACCI with pc-ACC (proto-calcite ACC) and $\mathrm{ACCII}$ with pv-ACC (proto-vaterite $\mathrm{ACC}$ ), respectively. These ACCs were precipitated from metastable solutions of calcium carbonate at different $\mathrm{pH}$ values by destabilization in excess ethanol.

TEM (Figure 1) reveals the ACCs as spherical particles with a diameter of approximately $20 \mathrm{~nm}$. Small-angle X-ray scattering (SAXS) data support this characteristic size
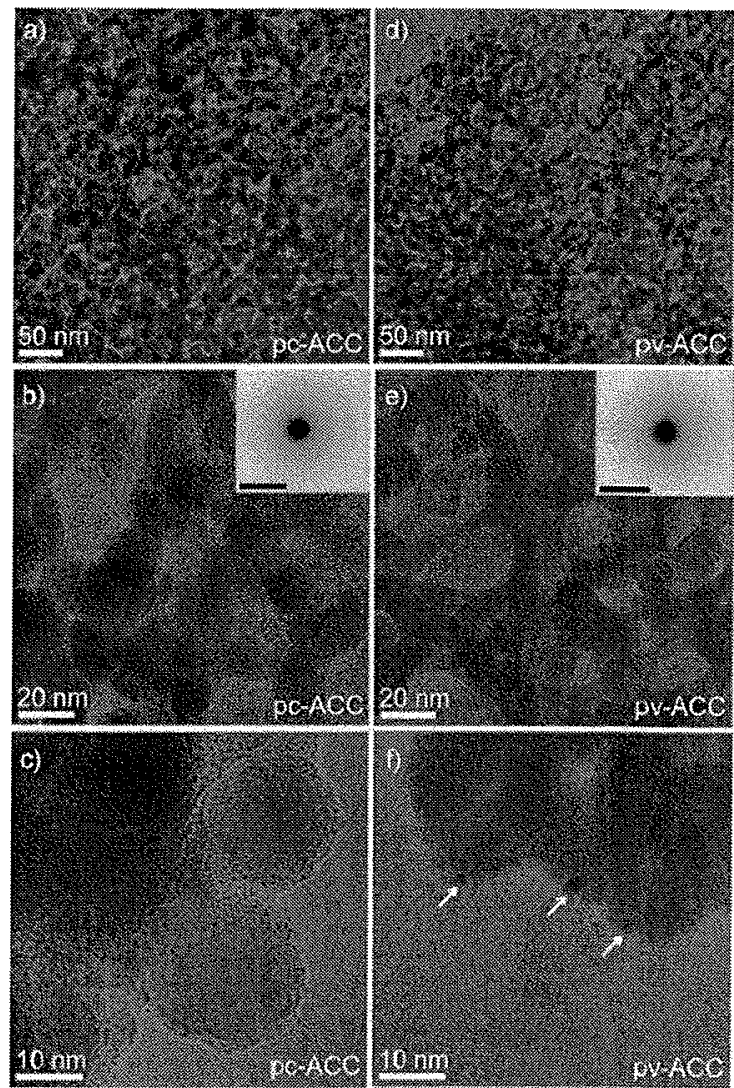

Figure 1. TEM images of $\mathrm{PC}-\mathrm{ACC}$ and $\mathrm{pv}-\mathrm{ACC}$ at various magnifications. Insets in (b) and (e) are selective area electron diffraction (SAED) patterns obtained from an area slightly larger than the particular image sections, SAED scale bars: $5 \mathrm{~nm}^{-1}$. SAED patterns are shown as negatives to make weak features clear. Arrows in ( $f$ ) indicate nanostructural features. 
(Supporting Information, Figure S1), which agrees with cryoTEM observations by Pouget et al. ${ }^{[14]}$ This characteristic particle size with a narrow distribution may relate to sizelimiting build-up of charge on the nascent particles. ${ }^{[23,24]}$ In the case of pc-ACC, the primary particles tend to form larger compact aggregates (Supporting Information, Figure S2). The amorphous character of the ACCs is shown by electron diffraction (SAED, insets in Figure $1 \mathrm{~b}$ and Figure 1e), and is further corroborated by $\mathrm{X}$-ray diffraction data (Supporting Information, Figure S3). We occasionally observe features (ca. $2 \mathrm{~nm}$ ) in TEM images (indicated with white arrows in Figure $1 \mathrm{f}$ ), which may relate to pre-nucleation clusters within primary particles of $\mathrm{ACC}$.

The ${ }^{13} \mathrm{C}$ magic-angle spinning (MAS) NMR spectra (Figure 2a) of calcite and vaterite comprise narrow peaks with Lorentzian shapes. Calcite is associated with a single
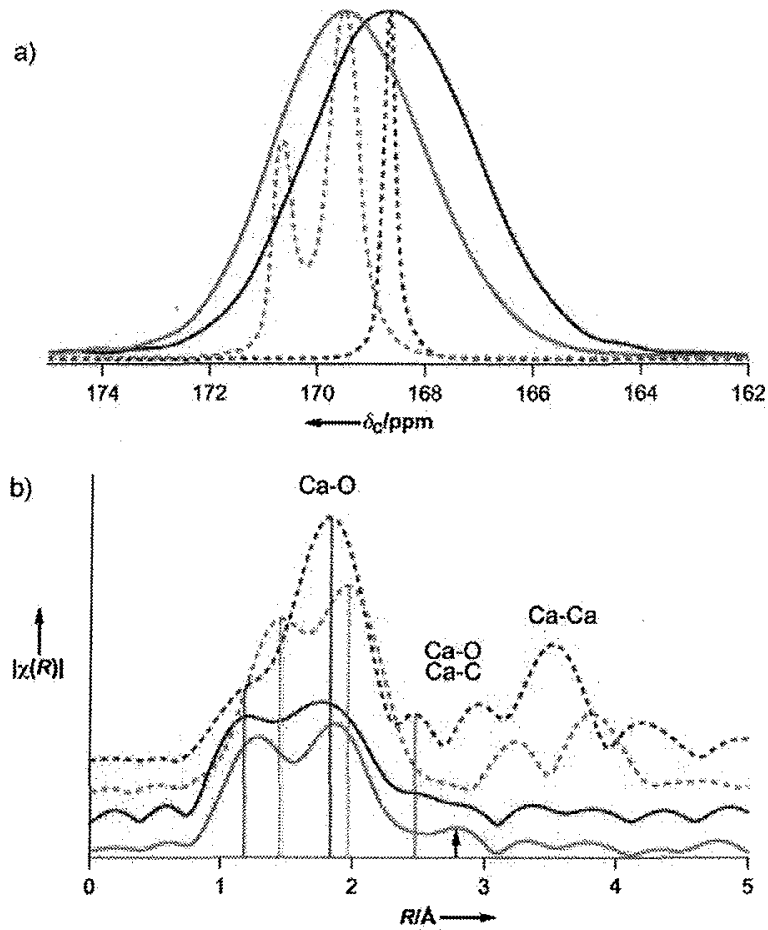

Figure 2. Spectra of calcite (dashed blue lines), vaterite (dashed red lines), $\mathrm{PC}-A C C$ (blue lines), and pv-ACC (red lines). a) ${ }^{13} \mathrm{C}$ solid-state NMR spectra recorded by single pulses at a magnetic field of $9.4 \mathrm{~T}$ and a MAS rate of $8.0 \mathrm{kHz}$. b) Fourier Transform of calcium $K$-edge EXAFS plotted in the $R$ space (lines as in (a)). The expected interactions of the first three coordination shells are indicated. The black arrow marks a peak, which may relate to the coordination of structural water. The vertical lines are a guide for the eye.

crystallographic site, giving rise to an NMR peak at $\delta_{\mathrm{C}}=$ $168.7 \mathrm{ppm}$. The two distinct carbonate ions of vaterite differ in their relative orientations, ${ }^{[2]}$ and give two NMR signals at $170.7 \mathrm{ppm}$ and $169.5 \mathrm{ppm}$ that are associated with relative integrals 1:3. The NMR parameters (Supporting Information, Table S1) accord well with previous reports. ${ }^{[21]}$ The pv-ACC and pc-ACC phases provide significantly broader Gaussianshaped NMR signals (Figure 2a); this reflects distributions of chemical shifts, as typically observed from amorphous materials. However, each peak maximum (i.e., the average ${ }^{1.3} \mathrm{C}$ chemical shift) from pc-ACC and pv-ACC is identical to that of calcite and the primary resonance of vaterite, respectively, which suggests closely related local carbonate environments in each crystalline modification and its amorphous counterpart. The very similar NMR peak-widths (of ca. $3.5 \mathrm{ppm}$ ) from the two amorphous phases imply similar extents of distributions around each respective average local structure.

The secondary NMR signal observed from vaterite $\left(\delta_{C}\right.$ $\approx 171 \mathrm{ppm}$ ), which becomes clearly visible during crystallization (Supporting Information, Section S2.1), is not revealed from the broad peak-shape of pv-ACC. As discussed thoroughly in the Supporting Information, Section \$2.1, the secondary vaterite-like site may be absent or significantly depleted within the structure of pv-ACC. However, we cannot exclude that the ${ }^{13} \mathrm{C}$ NMR chemical shifts of two potentially present and distinct carbonate ions in the pv-ACC structure are displaced slightly so as to produce a weighted chemical shift that incidentally coincides with the most intense ${ }^{13} \mathrm{C}$ NMR peak from vaterite. Indeed, IR data (see Supporting Information, Figure $\$ 4$ and Section $\$ 2.2$ ) provide evidence of two carbonate sites present in pv-ACC (but only one in pcACC) and further support the notion of distinct protocrystalline structures in the different ACCs. The IR data also confirms that the ACCs are free of organic additives.

The Fourier Transform of $\mathrm{Ca} K$-edge extended X-ray absorption fine structure (EXAFS) spectroscopy ( $R$-space Figure $2 \mathrm{~b}, k$-space Supporting Information, Figure S5) shows that the main peak for the first-coordination shell $(\mathrm{Ca}-\mathrm{O})$ is shifted to a higher $R$-value for vaterite $(R=1.96 \AA)$ compared to calcite $(R=1.81 \AA)$; this trend is reflected in the spectra of $\mathrm{pc}-\mathrm{ACC}$ and $\mathrm{pv}-\mathrm{ACC}$. The doublets arising from $\mathrm{Ca}-\mathrm{O}$ coordination are less defined in the spectra of calcite and $\mathrm{pc}-\mathrm{ACC}$ than in those of vaterite and pv-ACC. A shoulder in the spectrum of the pc-ACC $(R=2.5 \AA)$ correlates with a peak in the calcite spectrum, but not in the spectra of vaterite and pv-ACC. Quantitative EXAFS analyses (Supporting Information, Figure $\mathrm{S} 6$ and Section S2.3) indicate a slightly longer $\mathrm{Ca}-\mathrm{O}$ distance (by ca, $0.03 \AA$ ) in pv-ACC than in pcACC. An identical difference is found for vaterite and calcite. ${ }^{[26]}$ The evaluated $\mathrm{Ca}-\mathrm{O}$ distances and Debye-Waller factors generally accord with those reported for various ACC phases, whereas the corresponding EXAFS-derived coordination number of $N=2$ (first coordination shell) is rather low (Supporting Information, Table S3). Note that opposed to the $\mathrm{Ca}-\mathrm{O}$ distance, the value of $N$ is of limited accuracy owing to the mathematical properties of the EXAFS equation. ${ }^{[27]}$ Overall, EXAFS verifies similar local environments of calcium ions in $\mathrm{pc}-\mathrm{ACC} / \mathrm{calcite}$ and $\mathrm{pv}-\mathrm{ACC} / \mathrm{vaterite.}$

We argue that the peak in the EXAFS spectra marked with a black arrow in Figure $2 \mathrm{~b}$ may relate to water molecules coordinated to calcium ions in ACC. Indeed, thermogravimetric analysis suggests that both ACCs are stoichiometric $\mathrm{CaCO}_{3} \mathrm{H}_{2} \mathrm{O}$ (Supporting Information, Figure S12). The presence of structural water is corroborated by additional NMR experiments (Supporting Information, Section S2.1), and oxygen $K$-edge XANES (Supporting Information, Section S2.4) indicate distorted hydrogen bonding. ${ }^{[28]}$ Analyses 
of Ca $\mathrm{K}$ - and $L_{2,3}$-edge XANES spectra (Supporting Information, Section $\$ 2.4$ ) show that the calcium coordination in the ACCs is neither octahedral nor centrosymmetric, ${ }^{[29,30]}$ similar as in biogenic and "calcitic" ACC. ${ }^{[2]}$ We suggest structural water (coordinated to calcium ions) in pv-ACC and pc-ACC as an explanation of the low coordination numbers for calcium and deviations from certain symmetries.

Transformation of the proto-crystalline ACCs in pure water, or carbonate buffers of different $\mathrm{pH}$ value, generates both calcite and vaterite in varying relative amounts (Supporting Information, Figure S13 and Figure S14); crystallization by fast heating to $350^{\circ} \mathrm{C}$ generates pure-phase calcite regardless of the ACC used (Supporting Information, Figure S15), while MAS-induced crystallization produces mainly vaterite (Supporting Information, Section S2.1). Detailed investigations of these transformations are beyond the scope of this report, but the different crystallization tests indicate that kinetic factors, and competition between dissolutionrecrystallization and solid-state transformation need to be considered; these parameters are strongly influenced by mineral-additive interactions. ${ }^{[31,32]}$

In conclusion, we show proto-calcite and proto-vaterite features in additive-free ACC. The preparations of the two ACCs mainly differ in the pHvalue of the metastable solutions, which supports earlier claims that the structuring of amorphous intermediates relates to inherent features of pre-nucleation clusters of calcium carbonate and the underlying $\mathrm{pH}$-dependent equilibria. ${ }^{[15]}$ Consequently, proto-crystalline order is intrinsic to these ACCs; however, this structuring is only one factor influencing crystallization. Additive-free proto-crystalline ACCs appear to be of pivotal importance to further analyses of the processes involved in crystallization of calcium carbonates.

\section{Experimental Section}

In brief, $10 \mathrm{~mm}$ calcium chloride solution was slowly added to $10 \mathrm{~mm}$

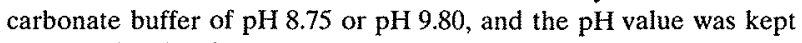
constant by titration. Before nucleation of $\mathrm{CaCO}_{3}$, the metastable solution was rapidly poured into an excess of ethanol, resulting in a mixture of over $80 \%(\mathrm{v} / \mathrm{v})$. The mixture was stirred for approximately $30 \mathrm{~min}$, left standing for approximately $2 \mathrm{~h}$, decanted, and the precipitate was centrifuged. Then, the precipitate was washed with pure ethanol, isopropanol, and acetone, and eventually dried. The synthesis with starting $\mathrm{pH} 8.75$ and $\mathrm{pH} 9.80$ yielded pc-ACC and pvACC, respectively. For a detailed experimental section, see the Supporting Information, Section S1.
[1] R. S. K. Lam, J. M. Charnock, A. Lennie, F. C. Meldrum, CrystEngComm 2007, 9, 1226-1236.

[2] Y. Politi, Y. Levi-Kalisman, S. Raz, F. Wilt, L. Addadi, S. Weiner, I. Sagi, Adv. Funct. Mater. 2006, 16, 1289-1298.

[3] B. Hasse, H. Ehrenberg, J. C. Marxen, W. Becker, M. Epple, Chem. Eur. J. 2000, 6, 3679-3685.

[4] Y. Politi, D. R. Batchelor, P. Zaslansky, B. F. Chmelka, J. C. Weaver, 1. Sagi, S. Weiner, L. Addadi, Chem. Mater. 2010, 22, $161-166$.

[5] L. B. Gower, Chem. Rev. 2008, 108, $4551-4627$.

[6] L. B. Gower, D. J. Odom, J. Cryst. Growth 2000, 210, 719-734.

[7] J. Aizenberg, L. Addadi, S. Weiner, G. Lambert, Adv. Mater. 1996, $8,222-226$.

[8] H. Cölfen, L. Qi, Chem. Eur. J. 2001, 7, 106-116.

[9] K. Gorna, M. Hund, M. Vučak, F. Gröhn, G. Wegner, Mater. Sci. Eng. A 2008, 477, 217-225.

[10] J. J. J. M. Donners, B. R. Heywood, E. W. Meijer, R. J. M. Nolte, C. Roman, A. P. H. J. Schenning, N. A. J. M. Sommerdijk, Chem. Commun. 2000, 1937-1938.

[11] S. Huang, K. Naka, Y. Chujo, Polym. J. 2008, 40, 154-162.

[12] A. Xu, Q. Yu, W. Dong, M. Antonietti, H. Cölfen, Adv. Mater. 2005, 17, 2217-2221.

[13] S. Raz, P. Hamilton, F. Wilt, S. Weiner, L. Addadi, Adv. Funct. Mater. 2003, 13, $480-486$

[14] E. M. Pouget, P. H. H. Bomans, J. A. C. M. Goos, P. M. Frederik, G. de With, N. A. J. M. Sommerdijk, Science 2009, 323, 14551458.

[15] D. Gebauer, A. Völkel, H. Cölfen, Science 2008, 322, 1819-1822.

[16] H. Lowenstam, S. Weiner, On Biomineralization, Oxford University Press, New York, 1989.

[17] J. R. Clarkson, T. J. Price, C. J. Adams, J. Chem. Soc. Faraday Trans. 1992, 88, 243

[18] J. Bolze, D. Pontoni, M. Ballauff, T. Narayanan, H. Cölfen, J. Colloid Interface Sci. 2004, 277, 84-94.

[19] Y. S. Han, G. Hadiko, M. Fuji, M. Takahashi, J. Eur. Ceram. Soc. 2006, 26, 843-847.

[20] A. L. Goodwin, F. M. Michel, B. L. Phillips, D. A. Keen, M. T. Dove, R. J. Reeder, Chem. Mater. 2010, 22, 3197-3205.

[21] F. M. Michel, J. MacDonald, J. Feng, B. L. Phillips, L. Ehm, C. Tarabrella, J. B. Parise, R. J. Reeder, Chem. Mater. 2008, 20 , 4720-4728.

[22] H. Nebel, M. Neumann, C. Mayer, M. Epple, Inorg. Chem. 2008, 47, 7874-7879.

[23] A. Navrotsky, Proc. Natl. Acad. Sci. USA 2004, 101, 1209612101.

[24] G. Bogush, M. Tracy, C. Zukoski, J. Non-Cryst. Solids 1988, 104, $95-106$.

[25] J. Wang, U. Becker, Am. Mineral. 2009, 94, 380-386.

[26] A. Becker, U. Bismayer, M. Epple, H. Fabritius, B. Hasse, J. Shi, A. Ziegler, Dalton Trans. 2003, 551-555.

[27] M. Epple, M. Panthöfer, R. Walther, H. J. Deiseroth, $Z$. Kristallogr. 2000, 215, 445-453.

[28] J. D. Smith, C. D. Cappa, K. R. Wilson, B. M. Messer, R. C. Cohen, R. J. Saykally, Science 2004, 306, 851-853.

[29] F. de Groot, J. Fuggle, B. Thole, G. Sawatzky, Phys. Rev. B 1990, $41,928-937$.

[30] G. Sarret, M. Isaure, M. A. Marcus, E. Harada, Y. Choi, S. Pairis, S. Fakra, A. Manceau, Can. J. Chem. 2007, 85, 738-746.

[31] D. Gebauer, A. Verch, H. G. Börner, H. Cölfen, Cryst. Growth Des. 2009, 9, 2398-2403.

[32] D. Gebauer, H. Cölfen, A. Verch, M. Antonietti, Adv. Mater. $2009,21,435-439$. 